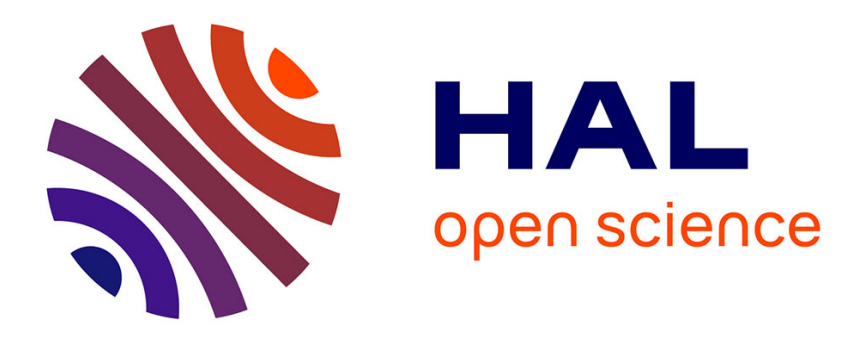

\title{
Premiers poèmes en prose: le spleen de la poésie
}

Nathalie Vincent-Munnia

\section{To cite this version:}

Nathalie Vincent-Munnia. Premiers poèmes en prose: le spleen de la poésie. Littérature, 1993, 91

(3), pp.3-11. 10.3406/litt.1993.2647 . hal-02076016

\section{HAL Id: hal-02076016 \\ https://hal.uca.fr/hal-02076016}

Submitted on 21 Mar 2019

HAL is a multi-disciplinary open access archive for the deposit and dissemination of scientific research documents, whether they are published or not. The documents may come from teaching and research institutions in France or abroad, or from public or private research centers.
L'archive ouverte pluridisciplinaire HAL, est destinée au dépôt et à la diffusion de documents scientifiques de niveau recherche, publiés ou non, émanant des établissements d'enseignement et de recherche français ou étrangers, des laboratoires publics ou privés. 


\section{Premiers poèmes en prose : le spleen de la poésie}

\section{Nathalie Vincent-Munnia}

\section{Abstract}

During the eighteenth century and the beginning of the nineteenth century, the relationship between prose and poetry is questioned in terms of a divergence between the "tyranny " of French versification, and what is felt to be «poetical ». The response was the invention of poetic prose ; then prose entered poetry with the prose poem. Close analysis reveals it to maintain, in its very form, the original « spleen » about poetry's poetry which led to poetic prose.

\section{Citer ce document / Cite this document :}

Vincent-Munnia Nathalie. Premiers poèmes en prose : le spleen de la poésie. In: Littérature, $n^{\circ} 91,1993$. Prose des poètes. pp. 3-11;

doi : https://doi.org/10.3406/litt.1993.2647

https://www.persee.fr/doc/litt_0047-4800_1993_num_91_3_2647

Fichier pdf généré le 16/01/2019 
Nathalie Vincent-Munnia, Université Lumière-Lyon 2

\section{PREMIERS POÈMES EN PROSE : LE SPLEEN DE LA POÉSIE}

\section{Il est de beaux poèmes sans vers, comme il est de beaux vers sans poésie ${ }^{1}$.}

Cette affirmation, renvoyant à une réalité littéraire qui paraît bien établie aujourd'hui, n'a pas un tel caractère d'évidence à l'époque de sa proclamation. Elle reflète au contraire une opinion alors encore paradoxale et au cœur des polémiques concernant la poésie au dix-huitième siècle : le rapport de la prose à la poésie.

Si ce siècle marque un tournant dans l'histoire de la poésie française par sa remise en cause de la domination exclusive du vers et par ses premières tentatives de prose poétique, il faudra attendre le dix-neuvième siècle pour que la prose fasse véritablement son entrée en poésie avec la création du poème en prose. On connaît bien sûr le rôle de Baudelaire dans l'invention - mais plus encore dans la diffusion - d'une " prose poétique, musicale sans rythme et sans rime ${ }^{2}$ "; mais la forme du poème en prose, en même temps que les prémices de sa reconnaissance générique, naissent bien avant Le Spleen de Paris, dès le début du siècle, avec certains textes de Xavier Forneret, Maurice de Guérin ou Aloysius Bertrand, voire Ludovic de Cailleux, Alphonse Rabbe ou Jules Lefèvre-Deumier.

La fin du dix-huitième et le début du dix-neuvième siècles constituent à cet égard une période essentielle de l'histoire de la poésie française : après l'insurrection contre les contraintes de la versification que traduit la naissance de la prose poétique, L'apparition d'une autre forme poétique, celle du poème en prose, reflète, elle, moins une nouvelle révolution qu'une seconde rupture esthétique qui témoigne d'un sentiment de

1. Abbé Jean-Baptiste Du Bos, Réflexions critiques sur la poéste et la peinture, Paris, J. Mariette, 1719, p. 679.

2. Charles Baudelaire, Dédicace - A Arsène Houssaye - du Spleen de Paris[éd. or. La Presse, 26 août 1862), Oetıvres complètes - I, Paris, Gallimard, 1975 (Bibliothèque de la Pléiade), p. 275. 
malaise et offre à la prose des pouvoirs neufs et des possibilités poétiques inédites : plus encore jeu sur ces possibilités poétiques que création d'une nouvelle poésie, le poème en prose et les premiers déjà - génère un discours poétique qui provoque une réflexion sur lui-même par sa propre dérision et renvoie la poésie à elle-même : un *spleen " de la poésie à l'image d'une poétique moderne...

Soumise à des normes invariables et à des codifications strictes, la versification française commence, dès le dix-huitième siècle, à apparaître comme bien contraignante. La pratique poétique se conforme certes encore largement à une esthétique néoclassique, respectant dans l'ensemble les règles édictées au dix-septième siècle; cette production est du reste abondante, largement diffusée ${ }^{3}$ et appréciée des lecteurs de l'époque. Mais certains théoriciens ou certains poètes dans des écrits théoriques attaquent cette poésie académique dans laquelle ils voient un obstacle à la manifestation spontanée, sincère de la sensibilité poétique : Fontenelle compare l'art de la poésie à * un tyran qui se plaît à gêner ses sujets, et qui ne veut pas qu'ils paraissent gênés ${ }^{4}$ "; D'Escherny quant à lui souligne que * les vers français sont pleins de ces contre-sens occasionnés par la tyrannie absurde de la mesure et de la rime 5 "; et Fénelon "avoue que nos plus grands poètes français gênés par les lois rigoureuses de notre versification, manquent en quelques endroits de ce degré de clarté parfaite ${ }^{6}$ "nécessaire selon lui à l'écriture poétique. La versification française apparaît ainsi sous les traits d'un tyran, cette figure si fréquemment critiquée par les philosophes, et subit donc une condamnation analogue.

Cette poésie néoclassique, essentiellement utilitaire et (ou) ornementale - descriptive, visant au didactisme et à la vulgarisation scientifique, ou circonstancielle, accompagnant tel événement de la vie quotidienne - exprime, dans des cadres (l'épître, l'ode...) et une rhétorique qui apparaissent usés, des

3. R. Navarri remarque qu'au dix-huitième siècle, le nombre des rééditions des œuvres poétiques est très souvent supérieur à celui des cuvres philosophiques ( $R$. Navarri, - La Poésie au dix-huitième siècle , in Michèle Duchet et J.M. Goulemot (dir.), Histotre littéraire de la France - Tome 5: 1715 - 1794 (1), Paris, éditions sociales, 1976, p. 201).

4. Bernard Le Bovier de Fontenelle, Sur la poéste en général [1742], Deuvres, Amsterdam, Aux dépens de la Compagnie, 1754, tome second, p. 133.

5. François-Louis comte d'Escherny, De la poésie et des vers, Mélanges de littérature, d'bistoire, de morale et de philasopbie, Paris, Bossange et Masson, et Schoell, 1811, tome 11, p. 229.

6. François de Salignac de La Motte Fénelon, - Lettre écrite à l'Académie française : sur l'éloquence, la poésie, l'Histoire, etc. - Projet d'une poétique :, Dialogues sur l'éloquence en général, et sur celle de la chaire en particulier avec une lettre écrite à l'Académie française [éd. or.], Paris, Florentin Delaulne, 1718, p. 310 (écrit en 1681-1686). 
sujets de convention. Excluant toute invention, elle est source d'ennui, voire d'un véritable " dégoût 7 ". Ces premières manifestations d'un * spleen * poétique conduisent certains auteurs, à la fin du dix-huitième siècle et dans les premières années du dix-neuvième, à choisir un nouvel instrument poétique et à forger une prose rythmée, imagée, susceptible d'exprimer un lyrisme et des sentiments personnels que le cadre du vers classique n'est plus capable de contenir. On attribue ainsi à Chateaubriand ou d'autres prosateurs une ambition proprement poétique; on considère même tels extraits d'Atala, les - Chansons indiennes " par exemple, isolés sous forme de fragments, comme de véritables poèmes, sans rimes ni mesures, mais dans une prose conservant un rythme riche d'effets de régularités, une prose que l'on nomme "nombreuse ", "harmonieuse " ou " cadencée ".

Mais cette prose, bien que regardée comme poétique, est le fait de prosateurs et forme le matériau d'écriture de récits. Or dans les premières décennies du dix-neuvième siècle, la prose est aussi utilisée par des auteurs écrivant par ailleurs en vers et dans des textes souvent plus courts et surtout autonomes, clos sur eux-mêmes et ayant leur unité propre, autre que purement narrative. Elle s'insère progressivement dans le cadre du poème.

En 1834 paraissent par exemple les Fragmens de LudovicMarie-Gabriel de Cailleux ${ }^{8}$. Ces textes sont écrits dans une prose proche, tant par son contenu que par son style, de celle des prosateurs préromantiques ${ }^{9}$ : l'inspiration religieuse domine mais on trouve également des "fragment[s] historique[s] " ou "dramatique[s] ", des morceaux de pur lyrisme, un " fragment fugitif " plus narratif et réaliste... La prose est donc pour une part au service de l'expression des sentiments personnels (religieux, amoureux...) et du lyrisme : dans "Une nuit sur les rochers d'Armorique 10" par exemple, l'évocation d'un amour ancien se fait chant, mêlant la description du paysage armoricain à la présence du " je * du poète et liant, par les

7. D'Escherny par exemple évoque le • dégoût profond que [lui] inspire la versification française - (De la Pó́sie et des vers, op. cit. p. 222).

8. Ludovic-Marie-Gabriel de Cailleux, Fragmens ou les Rapsodes religieux, Aix, Nicet, 1834. Ce recueil n'a jamais été réédité. Ludovic de Cailleux - dont les dates de naissance et de décès restent inconnues - est aussi l'auteur de ce qu'il nomme lui-même un - poème biblique en prose ", long texte écrit dans une prose imitée du verset biblique et constitué de quarante - testaments , lui non plus jamais réédité : Le Monde antédiluvien, poème biblique en prose, Paris, Comptoir des imprimeurs unis, Comon et Cie, 1845.

9. Dans ses Fragmens, Ludovic de Cailleux évoque du reste à plusieurs reprises directement Chateaubriand (. Lettre dédicatoire à ma mère - pp. 1-4, - Méditation . pp. 168-169, . Les Pyrénées - pp. 239-240,...) mais aussi allusivement Rousseau (- Rêveries d'un solitaire - pp. 152-160).

10. Ludovic de Cailleux, Fragmens, pp. 51-52. 
comparaisons et les métaphores notamment, la représentation de la nature à celle des êtres et de leurs sentiments, empreints de dolorisme :

\section{UNE NUIT sur les rochers d'Armorique}

Le papillon voltige de la marguerite au jasmin.

L'enfance a des cris de joie. Les jeunes filles se promènent en chantant sous les tilleuls et les marronniers. Le laboureur module des airs champêtres sur son chalumeau armoricain, vierges de Plèber et jolis enfans [sic] de Roscoff aux doux coquillages, chantez et priez. Et toi église de St Martin, tinte la cloche mélancolique à travers les bois.

Voilà les derniers feux du soleil, les derniers accens [sic] de la prière, de la joie et peut-être de la douleur.

Est-il quelque barde aux lèvres de feu qui chante sa Malvina sur sa lyre, caché dans les brouillards. Je ne suis pas ce barde. Mais à cette heure calme de la nuit, alors que l'on entend par intervalle les derniers bêlemens [sic] des troupeaux, que les jeunes fiancées viennent prier aux degrés de la croix solitaire dans le cimetière du village, que la brise agite les cordes de ma lyre, et que le souvenir luit dans mon cœur, je vais chanter. Voilà la nuit.

Je connaissais jadis une jeune Malvina chrétienne, une rose printanière, élevée sur les bords de l'océan.

Maint amant, abordant clandestinement le soir sur le rivage, l'appelait en vain. Moi seul je connaissais le signal du rendez-vous.

Elle venait alors.

L'innocence de ses soupirs enchantait mon cour. Ses cheveux ressemblaient à l'aile du corbeau sauvage. Elle était toujours pâle, mais pâle comme la feuille de la rose blanche. Elle souffrait et pleurait avec ceux qui pleuraient et souffraient. Son sein virginal se soulevait doucement sous les impressions de la jeunesse, ainsi que la vague azurée sous la brise matinale.

Son nom, je l'ai répété au sein des nuages. Les anges le connaissent, le pauvre l'a béni et moi je l'adore ; je le répéterai à mon heure dernière, si elle n'est pas là pour fermer ma paupière, elle qui a si souvent essuyé mes larmes.

Du point de vue stylistique et rythmique, la prose de Cailleux, tout comme la prose poétique du dix-huitième siècle, rappelle à maintes reprises le rythme du vers par des séries ou des régularités marquées : dans la quatrième strophe par exemple, deux unités syntagmatiques courtes ouvrent ("Je ne suis pas ce barde ", " Mais à cette heure calme de la nuit ") et ferment (* je vais chanter ", "voilà la nuit ") une succession de subordonnées temporelles plus longues répétant des schémas syntaxiques et rythmiques analogues; dans la dernière strophe, c'est la mesure de l'alexandrin qui rythme toutes les phrases, parfois renforcée par des rimes internes ("dernière "/ paupière"). Pourtant, audelà de ces analogies thématiques et rythmiques avec la prose poétique, les textes des Fragmens s'en distinguent par leur spécificité formelle: "Une nuit sur les rochers d'Armorique" débute par une description qui se développe en un véritable tableau champêtre; mais le flux bucolique est perturbé par une 
série de ruptures (succession de termes concrets désignant des référents singuliers ["le papillon "] et de termes génériques renvoyant à une notion abstraite [" l'enfance .] ; passage, dans la deuxième strophe et dans une même phrase, du présent à un impératif qui reprend, inversés, les sujets des deux phrases précédentes; rupture sémantique et rythmique à la fin de la troisième strophe; dans la strophe suivante, nouveau changement du thème et de la modalité de la phrase, puis de la personne grammaticale [avec l'intrusion du * je *]). De même, le deuxième mouvement du texte présente le * chant * du poète sous forme de souvenirs à l'imparfait ; mais, là encore, l'épanchcment lyrique est rompu, notamment par la division strophique et l'utilisation abondante de la parataxe; enfin, le flux rythmique de la dernière strophe est encore interrompu par les phénomènes d'éclatements et de chevauchements temporels. Tous ces effets de discontinuité brisent le déroulement purement narratif et/ou réflexif du texte et situent son unité à un niveau autre que celui de l'évolution d'une histoire, d'un personnage ou d'une pensée.

D'une manière plus générale, les premiers poètes en prose se caractérisent par une volonté sensible de resserrer leurs textes dans la forme du poème : par une présentation graphique (alinéas, blancs...) découpant le texte en courtes strophes, par des effets de "cadrage" (structures cycliques, présences de "refrains ", limitation du contexte...), par une unité d'effet... Ainsi, les pièces de Gaspard de la nuit sont des modèles de structure (reprenant une même division en couplets) et Aloysius Bertrand, dans ses "Instructions à $M$. le Metteur en pages * insiste sur la présentation de ses textes:

Règle générale. - Blanchir comme si le texte était de la poésie.

[.. ] M. le Metteur en pages remarquera que chaque pièce est divisée en quatre, cinq, six et sept alinéas ou couplets.

Il jettera de larges blancs entre ces couplets comme si c'étaient des strophes en vers ${ }^{11}$.

Le poème en prose se distingue donc de la prose poétique par cette structuration marquée en poème. Il s'ouvre aussi à des registres variés (alors que les prosateurs préromantiques visaient essentiellement au lyrisme) et surtout multiples : narratif, descriptif, voire historique ou réflexif. En témoignent les titres des différents " livres * de Gaspard de la nuit, reflétant soit une ambition descriptive - urbaine ("Le Vieux Paris") ou naturelle (*Silves *) ou encore plus plastique (*École flaman-

11. Aloysius Bertrand, - Instructions à $M$. le Metteur en pages ", [notes à l'intention de son éditeur supposé, Eugène Renduel], Gaspard de la nuitt, [éd. or. Iabitte, 1842], Paris, Gallimard, 1980 (collection Poésie), p. 301. 
de *) - , soit une dimension narrative, récits de rêves (* La Nuit et ses prestiges ") ou épisodes pittoresques (* Espagne et Italie *), soit enfin une inscription historique (* Les Chroniques *). De même, Alphonse Rabbe, dans la troisième partie de son Album d'un pessimiste ${ }^{12}$ — intitulée * Tristes loisirs * et comportant les textes dont l'esthétique annonce celle du poème en prose (et s'en rapproche le plus) - mêle de courts récits ("Le Naufrage *) et des descriptions d'objets (* La Pipe ", " Le Poignard ") mais qui intègrent elles aussi des paragraphes narratifs. La dimension descriptive se retrouve aussi dans Le Livre du promeneur ${ }^{13}$ de Jules Lefèvre-Deumier. Quant à Maurice de Guérin, ses deux célèbres poèmes en prose, Le Centaure et La Bacchante ${ }^{14}$, prennent la forme de récits de vie, tandis que ses poésies en vers se proposent plus fréquemment comme des "élégies" (au lyrisme plus directement marqué), des souvenirs (dans lesquels la narration cède alors le pas à la méditation sentimentale ou à l'évocation affective) ou des * rêverie[s] * (où, là encore, domine la subjectivité des émotions personnelles).

Le poème en prose constitue donc une nouvelle évolution par rapport à la prose poétique: si l'invention d'une prose poétique représente une rupture radicale par rapport au vers puisqu'elle permet au poétique de s'immiscer dans une forme d'expression jusque-là réservée à d'autres domaines, le poème en prose, lui, marque l'entrée réelle de textes en prose dans le champ de la poésie. Cette prose et ces textes, se constituant en poèmes au même titre que des poèmes en vers, acquièrent une pleine valeur poétique et peuvent alors tenter des registres considérés par la poétique du temps comme a-poétiques (tout en conservant une unité d'effet poétique). Le poème en prose, en entrant en poésie, élargit aussi le champ poétique et contribue donc à modifier cette poétique et ses normes.

Mais la virtualité la plus innovante qu'introduit la prose du poème en prose se situe au-delà du contenu stylistique, thématique ou générique du discours poétique. Elle touche au fondement même de ce langage poétique, précisément parce qu'elle n'est plus vers. Barbara Johnson ${ }^{15}$ a bien montré comment les Petits poèmes en prose, en insistant sur leur " statut de non-vers ",

12. Alphonse Rabbe, Guvres posthumes. Album d'un pessimiste, variétés littéraires, politiques, morales et pbilosophiques, Paris, librairie de Dumont, 1835-1836.

13. Jules Lefèvre-Deumier, Le Livre du Promeneur ou les mois et les jours, Paris, Amyot, 1854.

14. Georges-Pierre Maurice de Guérin, Reliquae, Paris, Didier, 1861. Le Centaure a d'abord été publié dans la Revue des detıx mondes du 15 mai 1840.

15. Barbara Johnson, Déftigurations du langage poétique, Paris, Flammarion, 1979 (collection Sciences humaines). 
convoquent un texte en vers dont ils mettent en évidence le fonctionnement figural et poétique par une * hyperbolisation du lyrisme * et une * mise à plat * des images et constituent ainsi une - littéralisation du langage . Le discours poétique, ne renvoyant plus qu'à son propre travail, se déconstruit lui-même ; la poésie entre, non pas déjà dans une ère du soupçon, mais dans une situation de duplicité où elle se dit en même temps qu'elle dit, dans un état de dérision où elle exprime une dissonance entre vision poétique et représentation de la réalité, à l'image du sentiment spleenétique qui transpose, dans l'ordre métaphysique, le choc vécu entre réel et idéal.

Les toutes premières réalisations d'une esthétique du poème en prose - les tout premiers poèmes en prose pourrait-on dire -, s'ils ne proposent pas de tels modèles de discours auto-référentiel, renvoient bien néanmoins par certains aspects à leur propre littérarité et comportent souvent des mécanismes d'intertextualité. Ainsi, de Cailleux propose-t-il une réécriture biblique, tandis que l'intertextualité chez Guérin est essentiellement mythologique, et chez Bertrand picturale et plastique ; celui-ci, dans le même temps, introduit, par son utilisation du grotesque, une fêlure dans le discours poétique qui défie alors la représentation et brouille les frontières entre réel et fantastique, comique et tragique.

Mais à cet égard, ce sont sans doute les poèmes de Xavier Forneret qui témoignent le mieux des possibilités offertes à la poésie par la prose, ceux du recueil Pièce de pièces Temps perdu ${ }^{16}$ notamment : : Un rêve c'est ", par exemple, révèle, dans un récit de rêve, toute une série de phénomènes de discontinuité (rythmique, syntaxique, logique, temporelle, énonciative...) ; un autre texte, "Le Diamant de l'herbe ", exploite abondamment des lieux communs romantiques mais les double de jeux d'écriture qui les déconstruisent de l'intérieur et mettent à jour les ficelles du discours poétique ; "Un désespoir " quant à lui fonctionne entièrement sur le mode déceptif :

\section{Un désespoir}

Je ne sais si l'abeille qui bourdonne et pique, si l'aigle qui trompette et déchire, si le gros chien qui hurle et mord, si le corbeau qui croasse et fouille, si le crocodile qui lamente et angoule, si l'éléphant qui barète et renverse, si l'épervier qui glapit et tiraille, si le hibou qui hue et crève les yeux, si le lion qui rugit et terrasse, si le perroquet qui cause et peut conduire à la mort, si le sanglier qui grommelle et déracine, si le serpent qui siffle et étreint, si enfin le tigre qui rauque et dévore, - je ne sais si ces

16. Xavier Forneret, Pièce de pièces Temps perdu [éd. or. Paris, Duverger, 1840], Oetuvres, Paris-Genève, Slatkine, 1980 (collection Ressources). 
quadrupèdes, reptiles et oiseaux, font plaies à ce qu'ils touchent, comme ce qu'on attend, et qui ne vient pas, nous jette au milieu du cœur l'amertume à gouttes, la déchirure au vif ;

Je ne sais : - mais un jeune homme rentrant chez lui, voulez-vous que je vous dise quelle preuve de désespoir il donna, de ce que l'heure d'un rendez-vous ne lui avait apporté que le son d'une cloche au lieu de son amante? - Eh bien! il était si heureux de toucher chaque nuit cette amante, - que, revenant à sa chambre, il passait toujours près de sa mère et l'embrassait, au risque de l'éveiller pour lui dire un mensonge. Cette nuit-là, il ne l'embrassa pas.

Le titre fait attendre le récit d'une histoire amoureuse; or la première strophe ne s'y rattache que par sa comparaison finale (" on attend ", "cœur", "l'amertume "...) qui, du reste, renvoie spéculairement au thème de l'attente déçue! De plus, cette première désillusion est renforcée par le fait que le point de comparaison entre le comparé (la douleur causée par une attente amoureuse déçue) et le comparant (les attaques animales) est très lointement motivé et ne porte que sur le deuxième terme de chaque membre de l'énumération (" pique ", "déchire ", "mord "... 17), le premier ("bourdonne ", "trompette ", " hurle «...) étant totalement inutile à l'élaboration de l'image ; il ne semble présent que pour allonger gratuitement l'énumération (et donc l'attente du lecteur) qui, du fait de sa longueur, se met alors à tourner à vide et ne fait plus que signaler les modalités de fonctionnement de la figure finale au lieu de les servir. La deuxième strophe, en introduisant des personnages (trois qui plus est) paraît enfin remplir les attentes du titre. Mais, là encore, tout est déconstruction de cette expectative: au niveau discursif, l'évolution narrative et le déroulement linéaire attendus sont supplantés par des chevauchements temporels ; en ce qui concerne l'énonciation, les interventions directes du narrateur et les modalisations viennent perturber le fil du récit ; sur le plan sémantique et rythmique enfin, la "preuve de désespoir ", enflée dans sa présentation, éclate dérisoirement dans le court syntagme final. Le travail narratif échoue donc, comme a échoué la représentation dans la - trop - longue énumération de la première strophe. Le récit attendu n'a pas lieu ou a lieu ailleurs (entre la mère et le fils ${ }^{18}$ ?) suscitant ainsi une nouvelle attente.

À la fois représentation et subversion de cette représentation, discours poétique et mise en lumière du fonctionnement poé-

17. En outre, ces termes tantôt appartiennent au vocabulaire technique, tantôt renvoient à des croyances populaires.

18. Les personnages de mères sont assez fréquents dans les textes de Forneret et ont surtout un rôle assez important et ambigu (par exemple dans * Un œil entre deux yeux * ou dans - Jeux de mère et d'enfant *). Une lecture psychanalytique de cette figure de la mère serait sans doute fructueuse. 
tique, le poème en prose est le lieu d'un déchirement. Forneret, du reste, exprime à plusieurs reprises ce sentiment de dissonance entre la quête poétique et l'aboutissement dans la réalité : "Tout est senti chez nous, sans pouvoir jamais bien en sortir ${ }^{19}$ "; ou encore : "L'auteur comprend la poésie comme il ne pourra jamais la faire. [...] Pour lui, dans ce monde, la poésie en tout, c'est son rêve. Quand il a écrit, c'est le réveil, et ce réveil l'accable. Il se trouvait bien, il se trouve mal ; il a tout senti, il n'a rien dit, puisqu'il a dit à sa manière ${ }^{20}$ *.

Produit d'un spleen métaphysique qui fait l'être duel, le poème en prose traduit en son sein même ce spleen : en même temps flux, rupture et jeu sur ces brisures, en même temps figuration, dé-figuration et défiguration, il dit le spleen d'une poésie qui ne peut plus dire que sur le mode de la dérision. Une poésie qui, dans la première moitié du dix-neuvième siècle, ne peut pas même se dire elle-même : les premiers poètes en prose qualifient leurs textes de "pièce de pièces" (Forneret), de "tristes loisirs" (Rabbe), de "bambochades" (Bertrand)... mais jamais de "poèmes en prose *. Il faudra attendre pour cela Baudelaire et ses Petits poèmes en prose; mais le spleen des premiers poèmes en prose demeurera dans les Petits poèmes en prose et, en se faisant parisien, il n'abandonnera pas sa dualité ni sa dissonance: la prose de ces poèmes ne doit-elle pas être " assez souple et assez heurtée pour s'adapter [à la fois] aux mouvements lyriques de l'âme, aux ondulations de la rêverie, aux soubresauts de la conscience ${ }^{21}$ "et la poésie n'est-elle pas en même temps "surnaturalisme et ironie ${ }^{22}$ "?

19. - L'Auteur ", hypotexte de Pièce de pièces Temps perdu, p. 147.

20. - Pour quelques passages de quelques-unes des vapeurs de ce livre ", hypotexte de Vapeurs ni vers ni prose léd. or. Paris, Duverger, 1838], CEtuves, pp. 82-83. 276.

21. Baudelaire, Dédicace - À Arsène Houssaye - du Spleen de Paris, op. cit. pp. 275-

22. Baudelaire, Fusées, op. cit., p. 658. 